\title{
Tratamiento de la neurocisticercosis: Revisión critica
}

\author{
Jorge Nogales-G aete ${ }^{1}$, Camilo Arriagada $\mathbf{R}^{2}$, Rodrigo Salinas $\mathrm{R}^{3}$. \\ Treatment of neurocysticercosis: $A$ \\ review
}

Neurocysticercosis (NCC) is the most common parasitic disease of the central nervous system. Several drugs, such as drugs against tapeworms, praziquantel or albendazole associated to corticosteroids, have been tested for the treatment of this condition. Although some have claimed the reduction or involution of cystic or granulomatous lesions, there is no consensus about the efficacy of these treatments. The natural evolution of the disease is not clear and this hampers the assessment of treatment effects. Moreover, there are no good imaging or clinical indicators that can predict the progression or spontaneous resolution of lesions, specially at the meningeal or ventricular compartment. Therefore, evidence based medicine does not have a definitive answer about the treatment, neither of seizures, the most common manifestation of NCC, or the varied and complex meningeal and ventricular involvement. This review includes experts opinions to give the clinician some clues for decision making in the treatment of NCC (Rev Méd Chile 2006; 134: 789-96).

(Key w ords: Albendazole; Neurocysticercosis; Praziquantel; Treatment)

Recibido el 30 de junio, 2005. Aceptado el 12 de septiembre, 2005.

${ }^{1}$ Servicio de Neurología, Hospital Barros Luco y Departamento de Neurología Sur, Facultad de Medicina, Universidad de Chile.

${ }^{2}$ Servicio de Neurología y Neurocirugía, Hospital del Trabajador de Santiago, ACHS.

${ }^{3}$ Unidad de Medicina Basada en la Evidencia, Ministerio de Salud de Chile.

L a neurocisticercosis (NCC), la parasitosis más frecuente del sistema nervioso central (SNC), consiste en la localización de la forma larvaria de la Taenia solium en el neuroeje propiamente tal o en los compartimientos ventrículo-meningo-licuorales del SNC.

Respecto del tratamiento, a pesar que durante estos últimos 25 años se ha atribuido a fármacos

Correspondencia a: Dr. Jorge Nogales-Gaete. Almirante Pastenes 249. Providencia Santiago. E mail: jnogales@ctcintemet.cl cesticidas, generalmente asociados a corticoides, el generar o favorecer la reducción del número 0 volumen de las lesiones quísticas y granulomatosas, no existe una conclusión definitiva de la efectividad de estos tratamientos ${ }^{1-11}$.

La persistencia de la controversia sobre el tratamiento de la NCC, se debe primero a la complejidad y diversidad de las entidades anátomo-clínicas que se agrupan bajo este diagnóstico, Tabla $1^{12}$. En segundo lugar, aún subyacen sin respuesta preguntas fundamentales para el tema: ¿cuál es la evolución natural de la enfermedad?, ¿de qué manera afecta el tratamiento, incluyendo 


\section{Tabla 1. Síndromes anatomoclínicos de la N C C}

1. Forma hemisférica o corticomeníngea (de tipo celuloso)

Forma quística viable: asintomática o con expresión de proceso expansivo

Forma encefalítica focal

Forma encefalítica polifocal masiva sincrónica, aguda y grave (tipo <encefalitis difusa») asincrónica, crónica y benigna (tipo epilepsia spolifocal»)

Forma pseudotumor cerebri

Forma granulomatosa calcificada, única o múltiple

2. Forma ventricular con síndrome de hidrocéfalo obstructivo (por quiste o ependimitis)

Hidrocefalia mantenida progresiva

Hidrocefalia transitoria

3. Formas meníngeas propiamente tales

Aguda o subaguda, meningítica o meningoencefalítica

Subaguda recurrencial

Crónica

Crónica pseudotumoral (focal persistente y progresiva)

4. Forma meníngea cerebrovascular

5. Formas espinales (meníngeas y parenquimatosas)

Modificada de Nogales-Gaete et $\mathrm{al}^{12}$.

cesticidas y corticoides, esta evolución natural?, en el manejo de las crisis epilépticas, la manifestación más frecuente de la NCC, ¿qué agrega el asociar tratamiento cesticida al tratamiento antiepiléptico? ¿qué factores determinan y qué indicadores (clínicos e imagenológicos) sugieren progresión, resolución espontánea completa o la formación de calcificaciones?, ¿cómo identificar, con la mayor seguridad, las condiciones clínicas en las cuales el tratamiento cesticida agrega el riesgo de agravar la condición del paciente? ${ }^{4}$. Antecedentes clínicopatológicos que sustentan estas inquietudes han sido revisados en extenso por los autores ${ }^{12-15}$.

\section{ESTUDIOS DE MEDICINA BASADA EN LA EVIDENCIA}

En los últimos años, hemos visto un esfuerzo creciente por diseñar mecanismos de búsqueda y análisis crítico de la información, sobre la eficacia de las intervenciones que entregamos a nuestros pacientes. Una de estas metodologías es aquella propuesta por la Medicina Basada en Evidencias (MBE), que busca integrar la mejor evidencia científica posible con la propia experiencia clínica y los valores y preferencias de los pacientes que buscan nuestra ayuda. La necesidad de disminuir al máximo la posibilidad de sesgos, de acuerdo a la $\mathrm{MBE}$, exige identificar -cuando existan- ensayos clínicos randomizados, o en su defecto, la mejor información científica disponible para ilustrar la decisión clínica ${ }^{16}$. Al aplicar esta metodología al tratamiento de la NCC, nos encontramos con una realidad que es común a muchas patologías propias de los países del Tercer Mundo: la investigación clínica de buena calidad metodológica que evalúa la eficacia de las intervenciones en estas áreas es escasa y difícil de obtener ${ }^{17}$. Los ensayos clínicos randomizados que estudian opciones terapéuticas en NCC son escasos y de pobre poder estadístico. Las recomendaciones terapéuticas que de ellos se desprenden son poco concluyentes, dejando la decisión clínica, en esta patología, fundamentalmente respaldada en la opinión de expertos $^{18,19}$. Esta situación se ve agravada por el empleo de desenlaces intermediarios de significado clínico controversial, como es el caso de la desaparición de los quistes con gran variabilidad de 
sustrato patológico de: encapsulación colágena, formación de calcificación o desaparición total ${ }^{13}$, en lugar de la mejoría clínica del paciente.

Salinas et al ${ }^{18,19}$, en sus metaanálisis para la Colaboración Cochrane, concluyen que aún faltan estudios, aleatorizados con grupo control, con suficiente número de casos para ser definitivos. La evaluación de los escasos ensayos clínicos randomizados existentes, sugieren la existencia de una positiva diferencia respecto del grupo tratado, en cuanto a la regresión o desaparición de las lesiones evaluadas imagenológicamente a los seis meses, pero en el límite de la significación estadística. En el caso de una epilepsia atribuible a una NCC, en tratamiento con anticonvulsivantes, no existe una conclusión, si el asociar tratamiento cisticida reduce el riesgo de recaída o recurrencia de crisis en los pacientes. En el manejo de la hidrocefalia, entidad con diversos sustratos clínico-patológicos, no existe respaldo sobre el beneficio del tratamiento cisticida. García et $\mathrm{al}^{11}$, han publicado recientemente un estudio que incluye 120 pacientes, distribuidos aleatoriamente y con doble ciego, en un grupo tratado con albendazol y dexametasona por 10 días y otro con placebo. Los resultados de este estudio muestran una disminución del número de lesiones en el grupo tratado con albendazol evaluados a los 6 meses. El desenlace principal estudiado en este ensayo fue el número de convulsiones con generalización, primaria o secundaria. Analizado el desenlace, el número fue significativamente menor en el grupo de pacientes tratado con albendazol. El número de pacientes libres de crisis durante el seguimiento, sin embargo, no fue diferente entre aquellos tratados con albendazol y aquellos que recibieron placebo: $56 \%$ y 51\%, respectivamente ${ }^{11}$. Esta discrepancia en los resultados, dependiendo del desenlace estudiado, hace difícil extraer una recomendación definitiva, que pueda ser utilizada al momento de tomar decisiones clínicas.

Mientras se solucionan las interrogantes ya mencionadas, y esperamos estudios con desenlaces que sean relevantes para el paciente, y que entreguen conclusiones con niveles de evidencia más categórica, es necesario tener una propuesta para aquellos casos que hoy requieren tratamiento. Con esta última finalidad, presentaremos y comentaremos en este artículo información sobre el tratamiento de la NCC.

\section{MOMENTOS Y MODALIDADES BÁSICAS EN EL TRATAMIENTO DE LA NCC}

El tratamiento disponible para la NCC, puede ser resumido, en seis grandes modalidades: 1.- Prevención y control sanitario. 2.- Fármacos cesticidas 0 antihelmínticos a los que se les atribuye la capacidad de causar la muerte de la larva y de la tenia. 3.- Corticoides y otros fármacos inmunodepresores, que disminuyen o evitan fenómenos inflamatorios (encefalíticos, meníngeos y vasculíticos) relacionados con la involución del quiste, sea espontánea o inducida. 4.- Fármacos antiepilépticos, que disminuyen la frecuencia o suprimen las crisis epilépticas. 5.- Diuréticos para manejar la hipertensión endocraneana. 6.- Procedimientos quirúrgicos, orientados a manejar la hipertensión endocraneana, la hidrocefalia y el efecto de masa de algunas lesiones ${ }^{10}$.

\section{PREVENCIÓN Y CONTROL}

En el control de esta parasitosis, la prevención es fundamental y para esto se requiere conocer el ciclo del parásito.

Ciclo del parásito. El hombre adquiere la teniasis intestinal al comer carne de cerdo mal cocida que se encuentra infectada con cisticercos. Esta larva está formada por un escólex o cabeza invaginada, contenida en una vesícula ovalada llena de líquido translúcido. Ésta es activada por el contenido del tubo digestivo, evaginando su escólex y fijándolo a la pared intestinal, desarrollándose posteriormente la forma adulta de la tenia.

El hombre, que hospeda la taenia adulta, puede eliminar con sus deposiciones proglótidas grávidas que contienen hasta 200.000 huevos embrionados, casi todos con capacidad infectante. Tanto el hombre como el cerdo, pueden ingerir los huevos a través de los alimentos contaminados con heces del portador. En el hombre también puede ocurrir autoinfección (ciclo ano-mano-boca o regurgitación de proglótidas al estómago), aunque en forma menos frecuente. Una vez digerida la pared externa del huevo, se activa el embrión e ingresa a la circulación sanguínea o linfática, luego de penetrar la pared intestinal. De esta manera, puede llegar a diversos tejidos del orga- 
nismo, incluyendo el SNC, donde desarrollará la forma larvaria $7,8,20,21$.

Considerando los elementos mencionados del ciclo teniasis-cisticerosis es crítico, primero, el fomento y educación en relación a la relevancia de la higiene personal y ambiental, incluyendo: buen lavado de manos antes de comer y después de ir al baño, consumo de alimentos bien lavados y cocidos, control sanitario de la crianza del cerdo y adecuado sistema de eliminación de excretas humanas, que evite la ingesta por el cerdo. Segundo, es fundamental la detección y tratamiento de todos los portadores de la tenia, con una búsqueda precautoria en los manipuladores de alimentos y en grupos de riesgo, como son: comedores de carne cruda de cerdos y contactos de portadores de $\mathrm{NCC}^{8,20,22-25}$.

Dentro de las estrategias de prevención a nivel porcino, hay que seguir con interés el desarrollo de la vacunación, aun cuando por el momento no sea una herramienta acreditada a nivel veterinario clínico $^{21,26,27}$. A nivel humano, Carpio ha recapitulado las limitantes inmunológicas y logísticas que hacen de la vacunación una alternativa poco probable en este momento ${ }^{21}$.

Como propuesta de control de teniasis, no exenta de controversia y riesgo, se ha planteado el tratamiento masivo con cesticidas de poblaciones con alta prevalencia de cisticercosis, lo que logra reducir el número de portadores de teniasis $\mathrm{y}$, secundariamente, disminuye la cisticercosis porci$\mathrm{na}^{4,20}$. Para el tratamiento de la teniasis por $\mathrm{T}$ solium, los fármacos disponibles son niclosamida y praziquantel, recomendándose el primero por no atravesar la barrera hemato-encefálica, evitando así posibles reacciones encefalíticas sorpresivas por vesículas cerebrales no diagnosticadas. La niclosamida se emplea vía oral en dosis única de 2 gramos $^{8}$.

\section{TRATAMIENTO DE LA NCC}

Respecto del tratamiento de la NCC, el único consenso que hay es que éste debe definirse caso a caso. Esto, atendiendo a las enormes diferencias clínicas y patológicas que representan las distintas modalidades de compromiso, con significativas variaciones de gravedad en una misma modalidad, determinadas por número, localización, ta- maño y estado de las lesiones, con gran diversidad de reacciones tisulares. Por eso, a continuación relacionaremos la información disponible con algunos acotados escenarios clínicos, recogiendo las orientaciones de una guía de consenso sobre tratamiento propuesta por un calificado grupo de expertos ${ }^{7}$.

Formas hemisféricas, parenquimatosas o córticomeníngeas

1. Calcificación única o múltiple, sin otro compromiso meningo-encefálico. La lesión puede considerarse como un hallazgo imagenológico respecto de la sintomatología que motivó el examen del paciente. Lo habitual es no realizar ningún tratamiento relacionado a la NCC y controlar la imagen al año 7,28 .

2. Lesiones igual a 1, pero con epilepsia activa coexistente, potencialmente atribuible a la NCC. Nuestra conducta es tratar la epilepsia, con los fármacos y por tiempos que están establecidos para esta entidad independiente de la NCC. Carpio ${ }^{21}$, plantea tratamientos antiepilépticos de un año si no hay recurrencia de crisis y de 1 a 2 años respecto de la última crisis, en caso que exista recurrencia. Respecto de la NCC, la conducta es controlar imagenológicamente al año. Si la epilepsia es refractaria al tratamiento, y no existe una buena explicación para esto (tipo de fármacos, adherencia, niveles, etc.), nosotros estudiamos el líquido céfalo-raquídeo (LCR), en búsqueda de algún componente meningítico, que lleve a redefinir la presunción de inactividad de la NCC.

3. Vesículas, tipo celuloso (con escólex), epicorticales o profundas, sin refuerzo por contraste en exámenes de imágenes, sin otro diagnóstico que NCC. Si la expresión clínica es una epilepsia, la conducta suele ser, tratar primero la epilepsia y luego plantearnos el tratamiento de la NCC, con antihelmínticos y corticoides. Si el número de lesiones es $<5$, no existe acuerdo respecto de la conducta. Algunos investigadores consideran que, dado que la mayoría de las lesiones probablemente evolucionará espontáneamente a la desaparición o calcificación en dos años, lo que corresponde es solo observar la evolución natural de la NCC ${ }^{7}$. Compartimos este criterio en aquellos casos cuando existe la posibilidad real y expedita de acceso a control 
neurológico y, además, cuando existe buen control de crisis; en caso contrario, nuestra opción es tratar con cesticidas y corticoides. El objetivo alude a sincronizar la muerte de los parásitos en un período con monitorización clínica y bajo tratamiento antiinflamatorio, de modo que se reduzca la reacción meningoencefálica focal y vascular. Por otro lado, esta opción da satisfacción a la hipótesis que la epilepsia responde mejor a tratamiento luego del uso de anticesticidas, a pesar de que los datos evaluados por la Colaboración Cochrane no fueron concluyentes al respecto ${ }^{21}$. Finalmente la monitorización imagenológica de las lesiones, ante tratamiento cesticida, primero de la respuesta inflamatoria y luego de la involutiva, proporciona elementos adicionales para el diagnóstico.

$\mathrm{Si}$ el número de lesiones es mayor a 5 y menor de 100 y su aspecto es vital, la propuesta sugerida por el comité de expertos es tratamiento con cesticida y corticoides, sobre 100 lesiones no se recomienda tratar con pesticidas por la potencial magnitud de la encefalitis ${ }^{7}$. En nuestra opinión, el riesgo de una crisis de hipertensión endocraneana marcada, generada por encefalitis múltiples, también es válido bajo 100 lesiones, por lo que la opción deberá evaluarse caso a caso, dependiendo de las características de las lesiones: involución, tamaño y ubicación de éstas.

4. Lesiones igual a 3, incluyendo algunas vesículas con refuerzo por contraste en exámenes de imágenes sugiriendo proceso involutivo. La conducta planteada es similar a la anterior? ${ }^{7}$.

Si se trata de una NCC con solo una lesión con signos de involución, denominada muchas veces como lesión captante única o lesión anular única por su aspecto en la tomografía computada de cerebro, la conducta suele ser monitorizar la evolución natural y tratar solo la epilepsia si ésta existe $^{7,29}$.

Cuando la epilepsia presenta mala respuesta a tratamiento, a pesar de los ajustes pertinentes en fármaco y dosis y si las lesiones son sincrónicas, acumuladas y con mucho edema persistente, el tratamiento con corticoides y cesticidas ayuda como elemento de diagnóstico diferencial. Si no hay cambios imagenológicos de las lesiones ante el tratamiento, debe extremarse el diagnóstico diferencial con otras enfermedades granulomatosas y tumorales, considerando incluso el empleo de una biopsia de la lesión.

Formas intraventriculares. El tratamiento de las formas intraventriculares se orienta también caso a caso, según el sustrato patogénico. Lo primero a definir es la presencia o riesgo de hidrocefalia aguda, y, por tanto, la pertinencia de una ventriculostomía de emergencia o la instalación de una derivativa, con o sin fenestración del septum, según la localización del cisticerco. Para la indicación de resección de quistes se considera: número, localización y estado evolutivo de las lesiones, complejidad clínica general del paciente, capacidades y experiencia del equipo neuroquirúrgico tratante en neurocirugía abierta 0 endoscópica ${ }^{7,30}$. Como criterios de indicación de escisión quirúrgica del quiste, podemos mencionar efecto de masa crítico, obstrucción ventricular, disfunción de la válvula y duda diagnóstica ${ }^{30}$. Para algunos autores, la observación en resonancia magnética de contraste en la pared vesicular, es un signo de mal pronóstico, independiente del tratamiento quirúrgico que se emplee, en cuanto traduce la posibilidad de un proceso de ependimitis que tenderá a la cronicidad con obstrucción o atrapamiento, independiente de la presencia de la vesícula ${ }^{31}$.

El tratamiento de las formas intraventriculares considera no solo opciones quirúrgicas, sino también tratamiento médico con cesticidas asociados a corticoides $5,30,32,33$ y antiepilépticos, cuando sea pertinente. Es importante mencionar que el tratamiento cesticida en las formas intraventriculares del cuarto o tercer ventrículo, debe realizarse sólo cuando el riesgo de una descompensación hidrocefálica aguda esté bajo control, mediante una derivativa. Es importante reflexionar respecto de las limitaciones de las evaluaciones de tratamientos de estas formas. Las variaciones en el número, volumen e involución de las lesiones, de las reacciones encapsulantes y de los caracteres de la eventual hidrocefalia, así como el escaso número de pacientes que tienen esta forma de presentación, hacen poco probable que se lleven a cabo ensayos clínicos randomizados con adecuada 
validez, que entreguen información que ayude a la decisión clínica.

Formas meníngeas racemosas. Al igual que en las situaciones anteriores, la elección del tratamiento es caso a caso. Existen numerosos reportes de tratamiento, en casos y series de casos, con cesticidas y corticoides a repetición, pero sin grupo control, que sugieren el beneficio del tratamiento para esta categoría, expresado en la reducción del tamaño de los quistes o del proceso meningítico ${ }^{6,34}$. A pesar de la falta de evidencia originada en ensayos clínicos randomizados, existe consenso en esta opción 7 . Algunos clínicos, como Cruz et $\mathrm{al}^{4}$, combinan curas múltiples con terapias prolongadas a veces por meses con cesticidas y corticoides.

Formas vasculíticas. El uso de cesticidas aumenta el componente inflamatorio y vasculítico, incrementando el riesgo de infarto, por lo que el tratamiento se basa en corticoides y, eventualmente, inmunosupresores ${ }^{35,36}$.

Formas intrarraquídeas, intra o extramedulares. En esta situación existe reconocimiento de la escasa experiencia existente y, en ese contexto, la recomendación es tratamiento quirúrgico combinado o no con cesticidas y corticoides ${ }^{7,37}$.

\section{ESQUEMAS DE USO DE FÁRMACOS CESTICIDAS Y CORTICOIDES}

Praziquantel (PZQ). La mayoría de los estudios que han empleado $\mathrm{PZQ}$, han usado esquemas de tratamiento de dos semanas con dosis entre 50 a $75 \mathrm{mg} / \mathrm{kg}$, con similar efectividad y seguridad $10,38,39$. En los últimos años se han publicado experiencias (quistes celulosos hemisféricos), con aparente eficacia, de un día de tratamiento con dosis total de $75 \mathrm{mg} / \mathrm{kg}$ dividida en tres dosis 25 $\mathrm{mg} / \mathrm{kg}$, asociando cimetidina y dieta hiperproteica para aumentar la absorción y el nivel plasmático del cesticida. El PZQ es, por lo general, bien tolerado y sus efectos adversos incluyen cefalea y molestias digestivas.

Albendazole (ALB). Los esquemas de tratamiento han tenido un desarrollo empírico y, en su mayoría, consideran un ciclo de $15 \mathrm{mg} / \mathrm{kg} /$ día por $7-28$ días $^{9}$, los que han mostrado similar efectividad, independiente de su duración ${ }^{39}$. En algunas formas, en especial en los quistes subaracnoideos gigantes ( $>5 \mathrm{~cm}$ de diámetro), se han repetido hasta cuatro ciclos ${ }^{6}$ y tratamientos prolongados han sido propiciados por $\mathrm{Cruz}$ et $\mathrm{al}^{4}$ en las formas meníngeas racemosas, especialmente con acúmulos pseudotumorales. Los efectos adversos son escasos. Sin embargo, tanto PZQ como ALB, al provocar degeneración y muerte del parásito, generan una reacción inflamatoria local, la que alcanza su mayor expresión entre el quinto y séptimo día de tratamiento. Dependiendo del efecto sumatorio de encefalitis focales y reacciones meningíticas, esto puede llegar a determinar hipertensión endocraneana, en magnitudes muy diversas, incluyendo eventuales formas graves y fatales.

Corticoides. Los esquemas de tratamiento son también empíricos y emplean preferentemente prednisona o betametasona oral. La dosis habitual de prednisona es de $1 \mathrm{mg} / \mathrm{kg}$-peso/día y la betametasona es de 12 a $16 \mathrm{mg} /$ día dividida en dos o tres dosis. La duración varía entre cuatro semanas hasta meses según la entidad en tratamiento. Con la intención de modular y reducir la respuesta inflamatoria por el uso de cesticidas, sea por la hipertensión endocraneana o por aumento de vasculitis, el empleo de los corticoides suele preceder en dos a cinco días al uso de cesticidas. Esquemas de uso prolongado de prednisona 50 mg tres veces a la semana por dos años, han sido planteados para disminuir la disfunción valvular en derivativas por hidrocefalias secundarias a aracnoiditis $^{40}$. 


\section{REFERENCIAS}

1. Brink G, Schenone H, Díaz V, Parra M, Corrales M. Neurocisticercosis: tratamiento con praziquantel. Estudio preliminar. Bol Chil Parasitol 1980; 35: 66-70.

2. Alarcón F, Escalante L, Dueñas G, Montalvo M, RomÁn M. Neurocysticercosis: short course of treatment with albendazole. Arch Neurol 1989; 46: 1231-6.

3. Escobedo F, Penagos P, Rodríguez J, Sotelo J. Albendazole therapy for neurocysticercosis. Arch Intern Med 1987; 147: 738-41.

4. Cruz-Ultreras I, Cruz M. Tratamiento médico de la neurocisticercosis. En: Arriagada C, Nogales-Gaete J, Apt W, eds. Neurocisticercosis. Santiago: ARRYNOG 1997; 287-98.

5. Proaño JV, Madrazo I, García L, García-Torres E, Correa D. Albendazole and praziquantel treatment in neurocysticercosis of the fourth ventricle. J Neurosurg 1997; 87: 29-33.

6. Proaño JV, Madrazo I, Avelar F, López-Félux B, Díaz G, GRJjalva I. Medical treatment for neurocysticercosis characterized by giant subarachnoid cysts. N Engl J Med 2001; 345: 879-85.

7. Garcia HH, Evans CA, Nash TE, Takayanagui OM, White AC JR, Botero D ET AL. Current consensus guidelines for treatment of neurocysticercosis. Clin Microbiol Rev 2002; 15: 747-56.

8. García $\mathrm{H}$, González A, Evans C, Gilman R. Taenia solium cysticercosis. Lancet 2003; 361: 547-56.

9. Kalra V, Dua T, Kumar V. Efficacy of albendazole and short-course dexamethasone treatment in children with 1 or 2 ring-enhancing lesions of neurocysticercosis: a randomized controlled trial. J Pediatr 2003; 143: 111-4.

10. NASH T. Human case management and treatment of cysticercosis. Acta Tropica 2003; 87: 61-9.

11. García HH, Pretell EJ, Gilman RH, Martínez SM, Moulton LH, Del Brutto OH et al. A trial of antiparasitic treatment to reduce the rate of seizures due to cerebral cysticercosis. N Engl J Med 2004; 350: 249-58.

12. Nogales-Gaete J, Arriagada C, González J. Síndromes anátomo-clínicos de la neurocisticercosis. En: Arriagada C, Nogales-Gaete J, Apt W, eds. Neurocisticercosis. Santiago: ARRYNOG 1997; 117-38.

13. Arriagada C, Nogales-Gaete J, Matus C. [Pathology of neurocysticercosis]. En: Arriagada C, Nogales-
Gaete J, Apt W, eds. Neurocisticercosis. Santiago: ARRYNOG 1997; 53-115.

14. Arriagada V, Arriagada C, Terra E, Manen R, Julo D. Imagen de resonancia magnética (IRM) en neurocisticercosis. En: Arriagada C, Nogales-Gaete J, Apt W, eds. Neurocisticercosis. Santiago: ARRYNOG 1997; 209-78.

15. Nogaies-Gaete J, Arriagada C, Carrasco X, Gonzáiez J, Chávez A. Neurocisticencosis como paradigma de las parasitosis del sistema nervioso central. En: Nogales-Gaete J, Donoso A, Verdugo R, eds. Tratado de Neurología Clínica. Santiago: Ediciones Sonepsyn - Editorial Universitaria 2005; 363-74.

16. Sackett D, Straus S, Richardson W, Rosenberg W, HAYNES R. Evidence Based Medicine: How to practice and teach EBM. $2^{\text {nd }}$ ed New York: Churchill Livingstone; 2000.

17. LeE K, MiLs A. Strengthening governance for global health research. BMJ 2000; 321: 775-6.

18. Salinas R, Counsell C, Prasad K, Gelband H, Garner P. Treating neurocysticercosis medically: a systematic review of randomized, controlled trials. Trop Med Int Health 1999; 4: 713-8.

19. Salinas R, PRasad K. Drugs for treating neurocysticercosis (tapeworm infection of the brain). Cochrane Database Syst Rev. 2000: CD000215.

20. Sarti E. Taeniosis y cisticercosis en México. En: Arriagada C, Nogales-Gaete J, Apt W, eds. Neurocisticercosis. Santiago: ARRYNOG 1997; 5-22.

21. Carpio A. Neurocysticercosis: an update. Lancet Infect Dis 2002; 2: 751-62.

22. Torres M, Pérez C, Galdámez E, Gabor M, Miranda C, Cofré X et al. Teniosis: serie clínica en 35 pacientes. Parasitol día 2001; 25: 55-9.

23. Gilman RH, Del Brutto OH, García HH, Martínez M. Prevalence of taeniosis among patients with neurocysticercosis is related to severity of infection. The Cysticercosis Working Group in Peru. Neurology 2000; 55: 1062.

24. Schantz PM, Moore AC, Munoz JL, Hartman BJ, Schaefer JA, Aron AM et al. Neurocysticercosis in an Orthodox Jewish community in New York City. N Engl J Med 1992; 327: 692-5.

25. Román G, Sotelo J, Del Brutto O, Fusser A, Dumas $\mathrm{M}$, WADIA N ET AL. A proposal to declare neurocysticercosis an international reportable disease. Bull World Health Organ 2000; 78: 399-406.

26. García H, González A, Gilman R. Diagnosis, treatment and control of Taenia solium cysticercosis. Curr Opin Infect Dis 2003; 16: 411-9. 
27. Piancarte A, Flisser A, Gauci CG, Lightowlers MW. Vaccination against Taenia solium cysticercosis in pigs using native and recombinant oncosphere antigens. Int J Parasitol 1999; 29: 643-7.

28. Ruey T, White Ac Jr. Management of neurocysticercosis. CNS Drugs 2003; 17: 577-91.

29. Padma MV, Behari M, Misra NK, Ahuja GK. Albendazole in single CT ring lesions in epilepsy. Neurology 1994; 44: 1344-6.

30. Madrazo I, Proaño J. Tratamiento quirúrgico de la neurocisticercosis. En: Arriagada C, Nogales-Gaete J, Apt W, eds. Neurocisticercosis. Santiago: ARRYNOG 1997; 299-321.

31. Citow J, Johnson P, Mc Bride D, Ammirati M. Imaging features and surgery-related outcomes in intraventricular neurocysticercosis. Neurosurg Focus (e-journal) 2002; 12: Disponible en URL http://www.medscape.com/viewpublication/ 65 toc?kol=12\&iss $=6$.

32. CuetTer A, ANDrews R. Intraventricular neurocysticercosis: 18 consecutive patients and review of the literature. Neurosurg Focus (e-journal) 2002; 12 (6): Disponible en URL http:// www.medscape.com/viewpublication/ 65 toc?kol=12\&iss $=6$.

33. Keley R, Duong D, Locke G. Characteristics of Ventricular Shunt Malfunctions among patients with Neurocysticercosis. Neurosurgery 2002; 50: 757-62.

34. Del Brutto O, Sotelo J. Albendazole therapy for subarachnoid and ventricular cysticerciosis. J Neurosurgery 1990; 62: 816-7.

35. BarinagarRementería F, Cantú C. Arteritis cerebral cisticercosa. En: Arriagada C, Nogales-Gaete J, Apt W, eds. Neurocisticercosis. Santiago: ARRYNOG 1997; 139-52.

36. Cantú C, BarinagarRementería F. Cerebrovascular complications of neurocysticercosis: Clinical and neuroimaging spectrum. Arch Neurol 1966; 53: 233-9.

37. Cow B, Moraes M, Carlotti C, Rubens H, Assirati J. Spinal cord cysticercosis: neurosurgical aspects. Neurosurg Focus (e-journal) 2002; 12 (6): Disponible en URL http://www.medscape.com/ viewpublication/ 65 _toc?vol $=12$ \&iss $=6$.

38. GARG RK. Medical management of neurocysticercosis. Neurol India 2001; 49: 329-37.

39. Pérez-López C, Isla-Guerrero A, Alvarez F, Budke M, Fernández-Miranda J, Paz J et al. Actualización en el tratamiento de la neurocisticercosis. Rev Neurol 2003; 36: 805-11.

40. Suastegui Román RA, Soto-Hernández JL, SOtelo J. Effects of prednisone on ventriculoperitoneal shunt function in hydrocephalus secondary to cysticercosis: a preliminary study. J Neurosurg 1996; 84: 629-33. 